\title{
ANÁLISIS
}

\section{Sesenta años de la República Popular: perspectivas locales sobre la evolución del Estado en China}

DOI: $10.32870 /$ mycp.v12i36.329

David S. G. Goodman ${ }^{1}$

\section{Resumen}

A unque la RPCh cumple 60 años de existencia en 2009, percepciones populares y hasta cierto punto académicas de su sistema político continúan condicionadas en exceso por la experiencia de sus primeros 30 años. El impacto socioeconómico de las políticas de las últimas tres décadas es sobradamente conocido, pero no el contexto en el que éstas se han producido. En particular, existe una tendencia a diferenciar entre un espectacular crecimiento económico y sus consecuencias, y la falta de cambio político. Mientras que claramente el Partido Comunista de China sigue en el poder, es igualmente obvio que la reforma económica ha ido de la mano de un gran cambio político. Estudios sobre el estado de transición a niveles locales

1. David S. G. Goodman es profesor de Política China en la Universidad de Sidney, donde también es director del Instituto de Ciencias Sociales. Estudió en la Universidad de Manchester (Política e Historia Moderna), en la Universidad de Pekín (Económicas) y en la Escuela de Estudios Orientales y Africanos de Londres (Chino y Política China). Sus investigaciones se han centrado en la política provincial de China, la historia del Partido Comunista de China y el cambio social y político en China desde 1900, especialmente a nivel local. En la actualidad está llevando a cabo estudios sobre la formación de élites locales en la China contemporánea (con las doctoras Beatriz Carrillo y Minglu Chen) y sobre aventureros coloniales alemanes en China entre 1870 y 1937 (con la doctora Yixu Lu). Su publicación más reciente es Los nuevos ricos en China: futuros dirigentes, vidas presentes (2008). ORCID http:// orcid.org/0000-0003-4965-6177 
ciertamente sugieren que el cambio ha sido significativo. Además, estos estudios locales también indican la necesidad de conceptualizar más lo que se entiende por Estado en China. La idea de Estado es un concepto demasiado general y un instrumento de análisis demasiado contundente, comparado con la investigación que considera los valores y la ideología del Estado, la base social del poder político, las estructuras y los procesos del sistema político, los responsables con autoridad para tomar decisiones, la burocracia y la administración, y las interacciones internacionales del Estado.

El Tercer Pleno del $11^{\circ}$ Comité Central del Partido Comunista de China (PCCh), celebrado en diciembre de 1978, se ha considerado desde hace tiempo como un momento decisivo en la historia de los 60 años de la República Popular de China. Éste fue el encuentro que condujo a tres décadas de creciente prosperidad y anunció el compromiso del partido del Estado con la reforma y la apertura al mundo. Las tasas de crecimiento anual del PIB han sido de 9-10\%, por lo que la economía total de la RPCh es actualmente la segunda más importante del mundo, intensificando el debate sobre cuándo igualará o superará a la economía de Estados Unidos, y sobre cómo el resto del mundo debería afrontar este fenómeno (Economy and Segal, 2009; Scissors, 2009).

Las consecuencias socioeconómicas de las decisiones del Tercer Pleno son bien conocidas. La economía de la RPCh ha sido esencialmente reestructurada, especialmente a través de la introducción de elementos de mercantilización en un sistema socialista de Estado (Huang 2008). La RPCh se ha convertido en un gran centro global de fabricación. En el proceso, aun cuando un sector sustancial de la economía continúa en manos del Estado, actualmente existen otros tipos de empresas que dominan en muchos de sus sectores. Al mismo tiempo, las economías esencialmente regionalizadas de la RPCh se han integrado más en las tres décadas mediante el desarrollo de la infraestructura de las comunicaciones y a través de mercados más amplios para la circulación de mano de obra y mercancía (Keng, 2001; Groenewold, 2008). Estos desarrollos económicos se han visto acompañados de un cambio social bastante considerable. La vivienda se ha privatizado en gran parte y el bienestar social se ha reconstituido casi totalmente. Se han movilizado nuevas clases de mano de obra migratoria y varias categorías de nuevos ricos han sido los causantes y los beneficiarios de gran parte de este cambio (Goodman, 2008a).

Considerar el Tercer Pleno como un momento decisivo en estos términos no es totalmente inapropiado, pero sigue habiendo lagunas en este relato aparentemente simple que pueden distorsionar la comprensión de las diná- 
micas de cambio. En primer lugar, aunque los resultados económicos de la RPCh han sido espectaculares desde 1978, no fueron desastrosos entre 1952 y 1978, a pesar de que a veces pareció lo contrario. Durante esos años su PIB creció en una media anual de $4.39 \%$, superando a Estados Unidos y a Europa en el mismo periodo (Maddison, 2007: 44). Ésta no es simplemente una cuestión de interés estadístico, sino que es importante para entender que la RPCh, como régimen que se modernizaba antes de 1978, había comenzado a producir beneficios sociales y económicos. De hecho, se puede considerar a la economía anterior a 1978 como los cimientos en los que se han basado los éxitos de la era de la reforma, especialmente porque, aunque el sistema anterior estableció una infraestructura de Estado básica, también tuvo muchas ineficiencias económicas inherentes.

Una segunda deficiencia al considerar al Tercer Pleno como únicamente el comienzo de una exitosa historia económica, es que refuerza ese mensaje tan común de que mientras la RPCh se ha reformado económicamente, el cambio político ha sido mínimo o inexistente. Dada la relativa estabilidad en política estratégica que ha caracterizado a la RPch desde 1978, ésta sería una conclusión importante que deberíamos intentar mantener. Durante sus primeros 29 años de existencia, la RPCh experimentó una extrema inestabilidad política, con un debate considerable dentro de la dirección del Pcch sobre la dirección estratégica general, y seis o siete grandes cambios (dependiendo de la precisión con que se cuenten) durante ese periodo (MacFarquhar, 1991).

No obstante, existe un grupo de entendidos en la materia que no sólo sostienen que el desarrollo político de la RPCh va a la zaga de los cambios en economía y sociedad, sino que (en palabras de Minxin Pei) "las características principales de un partido único de Estado leninista siguen manteniéndose esencialmente sin cambios" (Pei, 2006: 4; Mann, 2007; Perry, 2007). Incluso ha estado de moda contrastar las transiciones del socialismo de Estado de la antigua Unión Soviética y la RPch en estos términos, afirmándose que esta última había priorizado la reforma económica sobre el cambio político, en contraposición con la sucesora de la Unión Soviética, la Federación Rusa, que había enfatizado el movimiento hacia el pluralismo político (Gordon White, 1994; McFaul, 1997). Un resultado es que la RPCh, en la conciencia popular fuera de China, a menudo sigue caracterizada por la política y el sistema de Mao Zedong, aunque en la historia de los 60 años de la RPCh hace 33 que Mao murió y más de 30 años del Tercer Pleno. 
Por supuesto, aquellos que sostienen que la RPch permanece "esencialmente sin cambios" probablemente no sostendrán que no ha habido ningún cambio, y tampoco aquellos que buscan identificar los elementos del cambio político pueden negar que la RPCh continúa siendo un Estado de partido dominado por el PCCh, sin una abierta oposición política institucionalizada. El problema es que las afirmaciones sobre el Estado sin cambios en estos términos enmascaran tanto como revelan.

Ciertamente el Estado se ha visto a sí mismo en un periodo de transición. Éste era el significado de "reforma" en la era de la reforma articulada a finales de 1978, aunque el objetivo final es una democracia más socialista que liberal (Goodman, 1985). En particular, el Tercer Pleno anunció un cambio en la visión del Estado de partido único sobre la relación entre Estado y sociedad. Desde mediados de los años cincuenta hasta finales de 1978 el Estado se había visto a sí mismo desde algo similar a una perspectiva totalitaria. Tenía una visión unitaria y holística del Estado y de la sociedad, en la cual el Estado manejaba directamente la economía y la sociedad, y era el iniciador del cambio social y económico. Los cambios introducidos en el Tercer Pleno se diseñaron para crear un régimen autoritario más reconocible, donde el Estado regula la sociedad y la economía indirectamente, e interviene menos directamente en actividades sociales y económicas.

Uno de los resultados de estos cambios fue necesariamente que el Estado y la sociedad se diferenciaron más claramente en algunos aspectos, al menos en el sentido de que tanto por separado como en conjunto el Pcch y el gobierno llegaron a reconocer que no eran responsables de ciertas actividades y que éstas ni siquiera pertenecían a su ámbito. Todo un espectro de instituciones se ha asentado en el espacio político creado por estos cambios, incluyendo cámaras de comercio, ONG e incluso asociaciones informales que pueden describirse de manera aproximada como "organizaciones de derechos" que protegen, o su propio interés (por ejemplo, en discusiones sobre uso de agua o tierras) o asuntos más altruistas (como por ejemplo, aquellas que ofrecen protección y apoyo a personas afectadas por el viH/sida) (Mertha, 2008; Weng, 2006; O’Brien, 2008).

Por fuerza, dados los principios fundacionales y los métodos de funcionamiento de la RPCh (por mucho que estén en transición), los límites entre Estado y sociedad continúan sin estar definidos con claridad, cambian constantemente y son, en todo caso, enérgicamente combatidos. De hecho, hay evidencia de la última década de que, mientras que en los años ochenta y 
noventa hubo una distinción clara entre el Estado y la actividad económica, más recientemente el Estado ha comenzado a tener el control en relación con su capacidad para llevar las riendas de sus recursos y de sus empresas, especialmente a nivel local (Huang, 2008: 283; Pei, 2006). Al mismo tiempo, está claro que un "Estado local depredador" (Pei, 2006: 35) está todavía a distancia de un Estado centralista y totalitario.

\section{El problema del Estado}

Un problema clave en este contexto es que el "Estado" es un concepto difícil de convertir en algo operativo, sobre todo porque no es estrictamente comparable con una situación en la que un sistema de gobierno (el régimen) se diferencia claramente de esa particular expresión de autoridad que es actualmente responsable del liderazgo o del gobierno, como es más común en una democracia multipartidista. Esta falta de distinción operativa entre un gobierno específico y el régimen es, por supuesto, una característica común de los Estados de partido único. Es prácticamente imposible considerar cualquier movimiento que se aleje del dominio unipartidista como menos que un cambio fundamental en el Estado, y los Estados de partidos únicos han tenido siempre la tendencia en la práctica (aunque no en sus principios) a evitar las distinciones gobierno-régimen, especialmente por su orientación teleológica (Ionescu, 1967).

Investigaciones sobre el desarrollo de localidades concretas y estudios del nivel local más generales, sugieren que el Estado y la política en China han cambiado considerablemente desde comienzos de los años ochenta. Una señal fiable del cambio es la descentralización, que ha sido una característica de la era de la reforma. En la RPch se habló de descentralización con anterioridad, e incluso se implementó parcialmente a mediados de los años cincuenta y a comienzos de los sesenta (Solinger, 1993), pero no de una forma con tanto alcance que alterase el sistema. Ahora, sin embargo, como observa Pierre Landry, la RPCh probablemente se ha convertido en el sistema estatal más descentralizado del mundo, con un gasto gubernamental de $70 \%$ a niveles subnacionales. Cualquier duda sobre esta afirmación proviene no tanto de una valoración de la descentralización de China, como de la información a nivel mundial (Landry, 2008: 3). Además, en ese proceso la separación del gobierno de gran parte de la administración económica es tan fundamental como el traspaso de la toma de decisiones desde el centro hasta las provincias 
y las localidades de la jerarquía territorial administrativa (Montinola, Qian y Weingast, 1995).

Periodistas y académicos de fuera de China se centran inevitable y desproporcionadamente en Pekín y en la política a nivel nacional. Sin embargo, puede que los cambios en política y en el Estado se manifiesten de forma más obvia a niveles locales. Por supuesto, el término "local" en el uso político y administrativo en la RPCh cubre una serie de jurisdicciones, algunas de las cuales difícilmente se podrían calificar como "locales" en cualquier otra parte del mundo. Para el Estado de partido único de la RPCh, "local" es cualquier cosa por debajo del nivel nacional, incluyendo jurisdicciones a nivel provincial, que son equivalentes a los países de la Unión Europea en población y área, e incluyendo también a Shangai, que ahora podría reclamar ser una ciudad mundial al menos al mismo nivel que Pekín (Friedmann, 1986). El nivel provincial es local en la RPch, pero también lo son distritos, ciudades, condados, pueblos y aldeas de prefecturas de rango inferior. La descentralización del gasto gubernamental y la toma de decisiones económicas han dado libertad a la asignación de recursos, la regulación y los impuestos a niveles locales. También han dado más espacio para maniobrar a oficiales locales, que pueden verse a sí mismos más como líderes locales que como agentes del Estado central.

La creciente importancia de entender el cambio local, ha llevado desde 1990 a la publicación fuera de la RPch de un gran número de estudios locales y provinciales sobre cambios sociales y políticos (Jae Ho Cheng, 1995; Linda $\mathrm{Li}, 2006 \mathrm{a})$. El cambio local se describe y se analiza de manera explícita, aunque posiblemente la conclusión de más largo alcance de estos estudios es una que es mucho más implícita: la necesidad y los medios para conceptualizar más al "Estado" en China. Las investigaciones se ocupan principalmente del Estado en transición, aunque su definición no está clara. Esto no es ni indeseable ni una estrategia inviable para explicar el cambio. El Estado, obviamente, puede entenderse y es entendido de muchas formas diferentes. A veces se iguala a la estructura política de una sociedad y el ejercicio de una autoridad soberana en el territorio de dicha sociedad, y a veces a gobiernos específicos en esa área. Para los analistas políticos la noción de Estado es por lo general más específicamente una definición del sistema de gobierno en el que tiene lugar la política; para los economistas, normalmente es más una caja negra de toma de decisiones regulatorias o un actor económico.

La mayoría de las definiciones de Estado se remiten a dos desarrollos del concepto que se produjeron a comienzos del siglo xx. Uno es el desarrollo del 
concepto de "la idea de Estado" realizado por geógrafos, la identidad dominante de la organización política en cualquier territorio, para explicar cómo las sociedades mantenían las estructuras de la toma de decisiones revestidas de autoridad dentro de territorios concretos (Hartshorne, 1950). El otro es la definición de Weber del Estado como una estructura que se asegura el monopolio del legítimo uso de la violencia para mantener sus órdenes (Weber, 1997: 154). Operacionalizaciones más tardías de estas ideas, especialmente cuando se aplicaron a Estados comunistas de partido único, se han centrado más en la existencia y en las estructuras del Estado que en el ejercicio del poder estatal. Una definición del Estado en China que va poco más allá de la descripción del gobierno de un partido único, dice algo fundamental sobre la RPCh, pero está claramente limitada. Hace suposiciones sobre el ejercicio del poder del Estado, pero corre el riesgo de minimizar la investigación a cuestiones de legitimidad y autoridad, y a procesos de toma de decisiones e implementación de políticas. También corre el riesgo de ser demasiado estática y no identificar los aspectos dinámicos del cambio.

Un antídoto para estos problemas consiste en adaptar perspectivas recientes que enfatizan hasta qué punto el Estado es un proyecto construido por la interacción social (Migdal, 2001). En vez de entender al Estado y a la sociedad como entidades inherentemente separadas, y hasta cierto punto en conflicto, esta perspectiva se centra en cómo interactúan los dos en el ejercicio del poder estatal (Perry y Selden, 2000). Por eso la estrategia para entender el Estado es identificar los elementos que constituyen la articulación, las estructuras y el funcionamiento del poder estatal, además de las limitaciones del ejercicio del poder del Estado. Por necesidad, esto supone que el Estado ya no se ve como algo estático, sino en un proceso constante de cambio. No todo cambio consiste en la transformación violenta y dramática que normalmente se describe como revolución, no obstante, el cambio es constante (Wertheim, 1974).

Desde la perspectiva local es posible identificar seis elementos clave del Estado en transición. Aunque cada uno se presenta y se considera por separado, se solapan necesariamente y se refuerzan mutuamente para presentar un modelo del Estado. Identificar y entender estos diferentes elementos en la construcción de un Estado también ayuda a explicar la extensión del cambio en las últimas tres décadas.

Las amplias conceptualizaciones anteriores de "la idea de Estado" son un buen punto de partida para comenzar la tarea de identificar los diferentes elementos que determinan el ejercicio del poder del Estado. Los valores y las 
creencias que forman el ejercicio del poder político (ideología) justifican la existencia del Estado y la dirección de su política. Esta dimensión del Estado es particularmente notable e importante donde hay o ha habido un compromiso revolucionario, y donde hay una ideología explícita, como es el caso de un Estado comunista de partido único. Igualmente, una gran parte de la legitimidad de cualquier Estado reside en sus cimientos sociales (base social): de aquí la importancia en la era moderna de la emergencia de la idea del Estado-nación. Sin embargo, esto no significa que no pueda haber diferencias significativas en las interacciones del Estado con

La base social del Estado ha cambiado espectacularmente con la inclusión en el Pcch de los nuevos ricos, y en particular de los hombres de negocios. A nivel local está claro que el núcleo de la nación política se ha convertido en la alianza entre los cuadros del partido y los empresarios la nación política en su conjunto, y una clase dirigente de base más estrecha. Éste ha sido claramente el caso de la pasada experiencia de los Estados comunistas de partido único, donde la ciudadanía a menudo ha sido variable respecto a sus clases.

En un Estado comunista de partido único sería lógico esperar que la ideología influya profundamente, e incluso en ocasiones lo condicione en exceso en el sistema de gobierno (las reglas y normas formales e informales de funcionamiento del sistema político o régimen). Una de las formas en las que la RPCh ha comenzado a cambiar en la era de la reforma es que, incluso aceptando comentarios anteriores sobre las dificultades para diferenciar entre régimen y gobierno en un Estado de partido único, como el sistema se ha institucionalizado más después de Mao, los responsables con autoridad para tomar decisiones (gobierno) se distinguen ahora conceptualmente. Se podría incluso discutir que el separar el régimen del gobierno era en cierto sentido un fin primordial de las reformas dirigidas por Deng Xiaoping. A su vez, tanto gobierno como régimen han de diferenciarse de las estructuras administrativas (burocracia) que implementan la política y las decisiones del gobierno.

El elemento final que da forma al Estado en estos términos, son sus relaciones internacionales. Especialmente cuando, como en este caso, el Es- 
tado de partido único se ha comprometido con la "apertura", sería razonable esperar que las interacciones con fuentes de poder e influencia de más allá de sus fronteras territoriales (otros Estados, instituciones internacionales e intereses económicos globales) influyesen significativamente en el ejercicio de poder del Estado. Debido a razones de espacio, este elemento del ejercicio del poder del Estado no se explica en detalle aquí.

\section{Ideología}

La actual Constitución de la República Popular China (1982) proporciona cierta guía sobre la ideología oficial del Estado. Como ha venido ocurriendo desde la fundación de la RPCh en 1949, sigue habiendo dos elementos en la justificación de la idea de Estado. La RPCh reclama los milenios de historia, civilización y cultura chinas, además del destino de modernización socialista, consagrado en la redacción del pensamiento marxista-leninista de Mao Zedong. Antes de 1978 la relación entre estos dos elementos en la ideología del Estado era un poco difícil en ocasiones, y algunos aspectos de la cultura china se atacaban directamente. Por ejemplo, durante la Revolución Cultural los vestigios de la "cultura tradicional" se convirtieron en objetivo a erradicar, y tras caer en desgracia Lin Biao en 1971 se le criticó como a Confucio. Esta tendencia que tiene el Estado a atacar a sus aparentes antecedentes históricos, a pesar de atribuirse la continuidad del Estado chino desde antes de 1911 (y la sucesión de las dinastías chinas), sin mencionar 1949, es una paradoja muy bien entendida en el desarrollo del nacionalismo chino moderno. La RPCh, como régimen explícitamente revolucionario, hace tiempo que tiene dificultades para determinar qué puede y debe conservar de épocas anteriores, y qué se debería reemplazar (Guo, 2004).

En la era de la reforma, la perspectiva local de este factor determinante del poder del Estado sugiere claramente que el equilibrio entre el nacionalismo cultural y la modernización socialista se ha inclinado mucho hacia el primero. Aquí también existen paradojas, ya que la señal de nacionalismo cultural constituye realmente un mayor discurso de localismo. Antes de 1978, un discurso de localismo articulado por líderes locales se consideraba casi siempre causa de destitución, si no de otro castigo peor (Teiwes, 1966). En 1958 el gobernador de Shandong, Zhao Jianmin, fue expulsado después de que supuestamente dijera: "Soy natural de Shandong. Estoy con la gente y los cuadros de Shandong" (scmp, 1958: 24). Sin embargo, en los años noventa fue casi de rigueur que 
los líderes provinciales y locales adoptaran un discurso localista de desarrollo (Oakes, 2000). A diferencia de los sucesos de 1958 que se acaban de citar, el gobernador de Shanxi, Hu Fuguo, fue felicitado por decir:

Nací en Shanxi, crecí en Shanxi, he vivido y he trabajado en Shanxi durante 44 años. Shanxi es mi hogar, y el pueblo de Shanxi me educó como hijo de campesinos. Como dicen, es difícil cambiar las influencias del hogar, es difícil olvidar los sentimientos del hogar. Nunca he podido olvidar el afecto de la gente de mi hogar. Mi propio destino y el de mi hogar están firmemente unidos ( $\mathrm{Hu}$ Fuguo, 1996: 11).

Mientras que el localismo se veía como una oposición al Estado antes de 1978, desde la adopción de políticas de "reforma y apertura" se ha abandonado esa ecuación. En cambio, el localismo y el fomento de la identidad local se ven ahora como una fuerza positiva que permitirá que los recursos, y en especial los recursos humanos como los empresarios dedicados a desarrollar sus lugares de nacimiento (Carrillo, 2008; Minglu Chen, 2008), se movilicen totalmente para alcanzar los fines nacionales de modernización. La sanción ideológica de la mercantilización de la cultura local emergió debido a los sucesos de junio de 1989. Los dirigentes del PCch vieron la necesidad de reforzar sus credenciales nacionalistas, subrayando su compromiso con "la extensa y profunda cultura tradicional china”, una fórmula que fue más allá que cualquier afirmación de ese tipo desde principios de los años cincuenta para relacionar a la China contemporánea con su desarrollo anterior a 1949. En enero de 1990 el entonces ministro de Cultura procedió a desarrollar la idea de la "cultura tradicional" como equivalente de las culturas chinas locales y populares (Guo, 2004: 31). Desde 1992 en adelante, casi todas las jurisdicciones a nivel provincial, y muchas otras localidades de rango inferior comenzaron a desarrollar estrategias específicas de desarrollo cultural que tenían como objetivo el desarrollo de identidades locales patrocinadas por el Estado (Jing Wang, 2005). Sin embargo, a diferencia del pasado, las expresiones de identidad local se consideran dentro de una jerarquía de identificación que se extiende desde la aldea al condado, a la provincia y a China en su conjunto: el localismo es, en resumen, uno de los cimientos del nacionalismo. Al mismo tiempo, el fomento de la cultura local inclina la balanza significativamente, acentuando el ejercicio del poder del Estado (Goodman, 2002).

Además de estos cambios en el marco ideológico del Estado, otros autores han visto también unos planes menos explícitos y aparentemente neoliberales 
que se pueden encontrar en el sistema de valores y creencias del Estado contemporáneo y que contrastan claramente con los valores de la era anterior a 1978, y especialmente con los de la Revolución Cultural (Harvey, 2005: cap. 5). Lee y Zhu, en su estudio sobre la reforma de la vivienda de Guiyang, explican estos valores con base en: la economía neoclásica, la regulación de mercado que reemplaza la intervención del Estado, la distribución económica que favorece al capital, el autoritarismo moral que se centra en la familia nuclear, el libre comercio internacional, y la oposición a la mano de obra sindicada. Sostienen que el Estado chino ha dado un giro claramente neoliberal, en especial por permitir que el mercado determine la distribución de recursos de vivienda y por fomentar la integración de China en la economía global (Lee y Zhu, 2009). Oakes, en su debate sobre las estrategias del desarrollo de la cultura local en Guizhou, va más allá. El argumento está claro. La creación de riqueza ha sido el principal mensaje que ha transmitido el Estado. Al mismo tiempo, los gobiernos locales se han hecho más emprendedores por necesidad, ya que los fondos centrales no han ido a la par del ritmo del crecimiento de la población y de la economía. Especialmente en las partes más pobres de China, las prácticas culturales locales pueden convertirse en una fuente financiera importante para los gobiernos locales, con la ayuda y la movilización apropiadas. En términos que resonarán en otras partes del mundo que han experimentado un giro neoliberal, la aparente reducción de la influencia del Estado provocó de hecho la privatización del patrimonio cultural promovida por el gobierno: una relación más cercana entre el gobierno y el sector privado de lo que podría haber sugerido una adhesión a los principios mercantiles (Oakes, 2006).

\section{Base social}

Como se podría esperar, los cambios en la construcción de la base social del Estado y en el desarrollo de la nación política de China son tan espectaculares como los contrastes ideológicos que acabamos de describir. La base social del Estado ha cambiado espectacularmente con la inclusión en el Pcch de los nuevos ricos, y en particular de los hombres de negocios. A nivel local está claro que el núcleo de la nación política se ha convertido en la alianza entre los cuadros del partido y los empresarios. Al mismo tiempo, está claro que las filas de los políticamente activos se han ampliado incluso más, sobre todo con el desarrollo de políticas específicas. Las consecuencias de estos cambios son quizás más claras para la construcción de la base social del Estado, donde 
una mayor polarización amenaza con minar la extensa atracción social del PCCh, que para el desarrollo de la nación política.

El PCCh y, por añadidura, la RPCh, habían atraído a ciertas categorías sociales y habían discriminado casi constantemente a otras entre 1949 y 1978 , aunque con pequeñas variaciones, en especial durante la era de la Revolución Cultural. Esta atracción se produjo generalmente entre los campesinos y los trabajadores. Los privilegiados eran aquéllos cuyo origen de clase y procedencia de clase (términos técnicos en la clasificación universal) se consideraban intachables: las familias de aquellos que se habían dedicado en cuerpo y alma a la revolución comunista, gente de orígenes campesinos y trabajadores que habían avanzado mediante el mérito académico y de servicio al Estado (White, 1976). La riqueza se convirtió rápidamente en un objetivo de discriminación del Estado a partir de 1949. En primer lugar, la reforma agraria intentó abordar el problema de la desigualdad en el campo; después, a mediados de los años cincuenta una serie de campañas urbanas finalmente llevaron a la socialización de la industria y del comercio. Aunque hasta mediados de los años cincuenta el PCCh siempre había trabajado con los propietarios de las tierras y con los líderes de la industria y del comercio, los empresarios y los hombres de negocios habían sido excluidos de la militancia del PCch desde comienzos de los años cuarenta, al menos. Desde mediados de los años cincuenta en adelante, las familias de aquellos que habían sido terratenientes y de antiguos empresarios fueron excluidas de la nación política.

La emergencia de nuevas categorías sociales de nuevos ricos empresarios y profesionales ha sido un distintivo de las tres últimas décadas y siempre fue probable que desafiara la construcción de la base social del Estado (Dickson, 2003: 89). Las formas en las que surgieron nuevas empresas, a menudo a través del desarrollo de capital rural colectivo o de la reestructuración del sector estatal, significaron inevitablemente que a mediados de los años noventa muchos empresarios no sólo eran miembros del Pcch sino que también habían ocupado (y a veces continuaban ocupando) puestos en el Estado de partido único (Dickson, 2008: 60). También está claro que al mismo tiempo a muchos empresarios privados que habían sido militantes del Pcch o que no habían tenido ningún nexo de unión con el Estado de partido único se les presionaba para que se unieran al partido, e incluso para que ocuparan puestos dirigentes a pesar de haber sido formalmente excluidos. Además, también se les invitaba con frecuencia a participar en el funcionamiento del Estado de partido único de otras maneras, ocupando puestos como miembros de go- 
biernos y de agencias del gobierno, o como diputados en congresos populares (Goodman, 2008b: 33).

El entonces presidente de la RPCh y secretario general del PCCh, Jiang Zemin, expresó públicamente por primera vez la noción de que se debería incluir a los empresarios y a los hombres de negocios en la conceptualización de la nación política en 2000. Ésta fue la principal innovación de su teoría de la "triple representatividad", donde los hombres de negocios representaban los elementos "más avanzados" en la sociedad china, que Jiang abogaba por incorporar al desarrollo de China (Jiang, 2002).

La reincorporación de la clase capitalista es claramente uno de los actos simbólicos más importantes de todas las medidas de reforma adoptadas por el Estado, aunque sólo se deba al contraste con el ejercicio del poder estatal desde comienzos de los años cincuenta. En verdad no se puede subestimar el simbolismo de estos cambios, que enfatiza no sólo los papeles del empresario y de la empresa, sino también la importancia de la riqueza en sí misma. Se dice que el líder de una aldea de Ox Market Fort, en la provincia de Guizhou, emitió algunos comentarios sobre la riqueza y los dirigentes locales de una forma que anteriormente hubiera sido imposible sin severas consecuencias políticas:

Antes de la liberación, ésta era una aldea muy instruida. ¡Más de un tercio de los hogares eran de terratenientes! [...] Actualmente, Jiang Zemin y Deng Xiaoping consideran a los terratenientes los representantes avanzados del progreso económico. Deberíamos entenderlo. En mi opinión, no eran terratenientes, no eran explotadores. Eran personas que trabajaban mucho, ahorraban y hacían avanzar a la aldea a la vez que avanzaban ellos mismos (Oakes, 2006).

Más allá del simbolismo, la realidad del desarrollo local, como se repite en todos los estudios académicos, es que la autoridad y de hecho el ejercicio del poder estatal local se ejercen ahora a través de la comunidad de intereses entre cuadros y empresarios. Por ejemplo, Blecher y Shue describen el desarrollo del condado de Shulu (Hebei) basándose en esta alianza (Blecher y Shue, 1996), Unger y Chan explican el fenómeno en detalle en Guangdong (Unger y Chan, 1999), y Whiting en Jiangsu, el Shangai rural y Zhejiang (Whiting, 2001). Ray Yep se centra en esta relación como el hilo conductor principal de la industrialización rural en Zibo (Shandong) (Yep, 2003). Chih-hou Chen ha explicado con detalle cómo esta alianza afecta a los derechos de propiedad en el delta del Yangtsé y en Fujian (Chih-hou Chen, 2004). Quizás el estudio 
más completo es el de Jean Oi, que encontró evidencia de la estrecha participación de cuadros y empresarios en Pekín, Liaoning, Sichuan, Tianjin, Shandong, Guangdong, Henan, Hunan, Jiangsu y Zhejiang en entrevistas realizadas desde 1986 hasta 1996 (Oi, 1999). Oi y otros se han referido en ocasiones a este fenómeno como la emergencia del "corporativismo estatal local" (Oi, 1992).

Posiblemente debido a que se ha registrado una concentración en el ejercicio del poder estatal a nivel local, es también aparente que la nación política se ha extendido de otras maneras. Incluso sin la posibilidad de oposición al PCCh, ya sea formal o de otro tipo, ahora hay una mayor diversidad de actores políticos que antes de 1978. El cambio social y su complejidad, especialmente como resultado del crecimiento económico y su impacto en el medio y en el estilo de vida han producido, como ya se ha apuntado, una serie de grupos altruistas y de interés personal. Por ejemplo, Andrew Mertha ha escrito sobre cómo los opositores en contra de proyectos de construcción de presas a gran escala han pasado a formar parte del proceso de elaboración de la política hidroeléctrica (Mertha, 2008). En el examen del desarrollo de un movimiento en contra de la violencia doméstica contra las mujeres, Louise Edwards identifica elementos que están en activo sin peligro dentro del Estado de partido único (miembros de la Federación de Mujeres), pero también encuentra que existen otros movimientos que están tan alejados que ni siquiera constituyen una organización de ningún tipo, y que sólo se reúnen en determinados actos y eventos (Edwards, 2009).

Las consecuencias a largo plazo de estos cambios se desconocen. Estudios sobre una serie de áreas problemáticas muestran alguna evidencia de que el cambio en la base social del Estado y la configuración actual de la política que impulsa la creación de riqueza pueden amenazar la polarización social. Esta conclusión es muy clara en varios estudios sobre la reforma de la vivienda que se han llevado a cabo en una diversidad de lugares tan distantes como Guiyang (en el sudoeste) y Shenyang (en el nordeste) (Lee y Zhu, 2009; Tomba, 2008). Fuera de las fronteras de la RPCh, especialmente en Norteamérica, hace tiempo que se esperaba que el crecimiento de un sector privado de la economía y de empresarios de mentalidad independiente desembocara en presiones hacia un cambio político, o incluso en una democracia liberal. La evidencia de las investigaciones de aquellos que han estudiado en detalle a empresarios privados muestra justo lo contrario. Tanto Bruce Dickson como Kellee Tsai han destacado la dependencia del sector privado del Estado de partido único y el 
deseo del Pcch de mantener su estrecha relación con empresarios privados hasta el extremo de que el término "privado" se cuestiona en este contexto (Dickson, 2008; Kellee Tsai, 2007).

\section{El régimen}

Está muy claro que la RPch continúa siendo un Estado de partido único, que es el PCch. Muchas de las estructuras de gobierno previas a la reforma continúan necesariamente incluyendo la preeminencia del Buró Político del PCCh y sus unidades subordinadas en la toma de decisiones autorizadas. Sin embargo, ha habido un cambio significativo en el sistema de gobierno. La conceptualización y la operacionalización de las relaciones entre Estado y sociedad han cambiado, como también lo ha hecho el control por parte del PCch de las actividades del gobierno y, como ya se ha comentado, la distribución espacial del poder.

Una de las características más importantes de muchas opiniones eruditas sobre la China anterior a la reforma, fue la presunción de que existía una oposición entre el Estado y la sociedad. En cuanto al Pcch, adoptó una actitud ligeramente distinta al pensar que podría y debería hablar por la sociedad y controlarla al mismo tiempo. La era de la reforma ha cambiado ambos puntos de vista sobre la relación entre el Estado y la sociedad. La mercantilización en general, y la comercialización del bienestar en particular, han situado el ejercicio del poder del Estado en una relación con la sociedad diferente de la que se tenía anteriormente. En su punto más extremo, estos cambios han significado que el Estado ya no es el responsable directo ante la mayoría de la población urbana, ni tampoco lo es indirectamente ante la población rural de las oportunidades de vida individuales de cada persona. De nuevo, el desarrollo de la reforma de la vivienda es un área donde este hecho se demuestra claramente, aunque se da de igual manera en el caso de los recursos de educación y de asistencia sanitaria (Lee y Zhu, 2009; Duckett y Carrillo, 2009). Además, como se ha comentado antes de manera general, y respecto al desarrollo de la base social, mientras el Estado continúa siendo un actor político por derecho propio hasta cierto punto, el sistema de gobierno ahora también permite que intereses parciales participen en el proceso político de maneras inimaginables tiempo atrás. Desde un punto de vista externo, no siempre se puede suponer que el Estado y la sociedad estén contrapuestos. Desde un punto de vista interno, se pueden escuchar otras opiniones en una 
especie de proceso de lobby junto al PCCh, aun cuando este último pueda seguir llevando la voz cantante en ocasiones y continuar tomando las decisiones (Perry y Selden, 2000; O’Brien, 2008).

Sería un error considerar la emergencia de intereses sociales, de ONG o incluso de intereses locales en oposición a dictados centrales como la emergencia de cualquier tipo de pluralismo político. El PCCh sanciona gran parte para asegurar una mayor eficiencia del socialismo, más que por una creencia en la virtud de la diversidad pluralista (Goodman, 1985). Al mismo tiempo, las formas en las que el Pcch ejerce su control han cambiado claramente. En el pasado, el control del PCch sobre los niveles inferiores de la estructura del Estado se aseguraba por medio de un estricto control de nombramientos en los puestos de dirección y creando temor al considerar las expresiones de interés local un crimen político de localismo. En las reformas de los últimos 30 años, en primer lugar, el número de cargos de dirección sujetos a nombramiento se redujo drásticamente y, como ya se ha mencionado, el Pcch ha fomentado el desarrollo de los discursos de localismo mediante el incremento de capital simbólico que puede ser aprovechado por el bien de la creación de riqueza. Por supuesto, el problema del control sobre los cuadros locales continúa y se ha resuelto asegurando requisitos más exigentes para entrar, tanto políticos como técnicos, y cantidades considerables de formación profesional y desarrollo profesional. Landry, en su estudio sobre las élites en Jiangsu, pone de relieve este punto y cita los comentarios de Deng Xiaoping en su Viaje de inspección al sur en relación con que "Debemos educar [...] Si surge algún problema en China, surgirá desde dentro del Partido Comunista [...] Debemos prestar atención a la formación de las personas" (Landry, 2008: 257).

El papel de la descentralización en la alteración esencial del sistema de gobierno en China ha sido una característica clave de la era de la reforma. Hay quienes critican que la descentralización continúa incompleta, y los problemas que esto conlleva, especialmente el surgimiento de una mayor corrupción (Gong, 2006). De cualquier forma, incluso hasta la fecha el alcance del cambio ha sido notable. Los gobiernos locales han obtenido más control sobre sus propios procesos presupuestarios, la recaudación de impuestos y la asignación de recursos, las empresas estatales e incluso sobre su participación en comercio exterior e inversiones extranjeras. Mientras que esto muestra un escenario en el que (desde el punto de vista del PCch) la formación de los cuadros puede ser más importante que nunca, y especialmente para no poner en peligro el mantenimiento de la autoridad política local del Pcch, puede 
haber resultados incluso más espectaculares e indeseados en los niveles más bajos del ejercicio del poder del Estado. Las presiones descentralizadoras, sobre todo en las regiones más pobres, pueden llegar a dejar a los condados, a los pueblos y a las aldeas luchando por el control de una base de recursos limitados. Además, las políticas de desarrollo regional en los últimos 20 años, que se han diseñado para mitigar los peores efectos del desarrollo desigual mediante la fundación de "ciudades regiones", condados dirigidos por ciudades y casos similares (todos ellos muy comunes a lo largo de la RPCh) parecen correr el riesgo de trasladar simplemente el control de recursos rurales a gobiernos urbanos (Hsing, 2006).

\section{Gobierno}

Estos cambios fundamentales del gobierno, y en particular la introducción de la descentralización, han tenido un profundo impacto en la localización $y$ en el funcionamiento del gobierno. Aunque normalmente se habla de la jerarquía administrativa territorial de la RPch en términos de centro y de localidad, se puede analizar de forma más útil en términos de tres niveles básicos de gobierno: central, provincial y local. El nivel provincial de jurisdicción incluye municipios directamente subordinados al Consejo de Estado (Pekín, Shangai, Tianjin y Chongqing) y las regiones autónomas (Xinjiang uigur, Xizang tibetano, Guangxi zhuang, Ningxia hui y Mongolia Interior). Los niveles locales incluyen condados y condados con nivel de ciudad, pueblos y aldeas. Con anterioridad a los años ochenta, de éstos sólo el nivel de condado se consideraba como parte del sistema formal de gobierno.

La descentralización en la era de la reforma ha tenido tres diferentes consecuencias en el funcionamiento del gobierno, concentradas todas ellas en los niveles más locales. Una gran parte de la toma de decisiones en materia de economía se ha localizado; los dirigentes locales, tanto en pueblos y aldeas como a nivel de condado, se han convertido en actores económicos por derecho propio y han dejado de ser simples agentes estatales, y el Pcch no parece funcionar a nivel local de la misma forma que lo hacía anteriormente. La esencia de estos cambios consiste en que el gobierno local y los dirigentes del gobierno local parecen ejercer mucha más autoridad ahora por méritos propios que antes.

No es difícil encontrar ejemplos de las formas en las que los cambios de la era de la reforma, y en particular la descentralización, han tenido como 
resultado que una mayor parte de la toma de decisiones significativa tenga lugar a nivel local. Una gran variedad de estudios han detallado cómo diferentes aspectos del desarrollo se planean y se ejecutan localmente. Gong detalla cómo la descentralización de la toma de decisiones económicas ha dado libertad a la asignación de recursos, las licencias, las inversiones y los impuestos a niveles locales (Gong, 2006). Hsing analiza la mayor capacidad de las localidades para participar en los procesos de gestión de la tierra, que sin duda ha sido crucial en el desarrollo industrial y de propiedades inmuebles (Hsing, 2006). Lee y Zhu tratan el tema del experimento local con la reforma de la vivienda (Lee y Zhu, 2009), y Oakes detalla cómo las localidades tienen la capacidad de elegir direcciones y situaciones específicas para sus estrategias individuales de desarrollo cultural (Oakes, 2006). Esto ha significado de manera inevitable que la práctica de la implementación de políticas difiera localmente, y en ocasiones en forma sustancial (Chih-jou Chen, 2004; Jae Ho Cheng, 1999; Jae Ho Cheng, 2000).

Por muy importante que pudiera ser este grado de descentralización por derecho propio, el proceso imperfecto de descentralización ha significado que los dirigentes locales se han convertido en actores económicos significativos y han dejado de ser simples agentes locales del Estado. A veces esta característica del comportamiento de los cuadros locales ha llevado a que se los describa como agentes del Estado local (Oi, 1995). Una descentralización más efectiva se concentraría en desconcentrar el poder a nivel local, y trataría de aumentar su responsabilidad y su representación (Gong y Chen, 1994). Mientras que lo primero ha sido claramente parte de la descentralización de la RPCh de la era de la reforma (al menos hasta el extremo de separar gobierno y funciones y funcionamiento del partido), el aumento de la responsabilidad y la representación han sido considerablemente más débiles. Un resultado es que los cuadros locales han sido capaces de desarrollar no sólo ingresos adicionales a los presupuestos (que el gobierno central sanciona hasta cierto punto fuera del proceso de planificación formal), sino también ingresos extrapresupuestarios (muchos de ellos desconocidos en altos niveles del gobierno). Estos recursos permiten que los funcionarios locales vayan más allá de los límites del presupuesto formal, que son un gran mecanismo de control directo a disposición de los niveles más altos del Estado (Gong, 2006).

Con las presiones políticas y económicas a las que se enfrentan para producir un crecimiento económico, los cuadros locales actúan con efectividad para mantener y desarrollar los intereses locales. Las campañas políticas que 
caracterizaron la administración de localidades antes de 1979 han desaparecido prácticamente. Su interés principal ahora es el desarrollo de la economía local. Además, aunque raramente poseen empresas propias cuando están ejerciendo su cargo, son importantes intermediarios y ofrecen su influencia y su ayuda. Como señala Gong, éste es un cambio importante en el gobierno ya que ahora, al contrario de años anteriores de la RPCh, el gobierno central tiene que "depender mucho de sus 'agentes' locales para gobernar en una estructura de poder cada vez más celular” (Gong, 2006). Éste es un proceso que a menudo produce un nivel de frustración considerablemente más alto en su capacidad de intervenir de modo significativo a nivel local (Hsing, 2006).

De alguna forma estas observaciones sugieren que el Estado de partido único puede estar comenzando a desmoronarse, por lo menos en las localidades. Aunque el PCch continúa central en la política a niveles centrales y provinciales, a niveles locales, aunque el Partido sigue existiendo, parece que está mucho más alejado del ejercicio del poder por derecho propio que a niveles más altos del sistema. Mientras que el Pcch está muy presente cuando se tratan temas de la política de la RPch o de dirección estratégica ya sea central o provincialmente, en el examen de estudios realizados en localidades específicas el pcch o lleva una sombría existencia o su presencia es inexistente. Como ya se ha mencionado, un mejor enfoque del análisis sería la comunidad de interés desarrollada entre los cuadros del partido único locales y los empresarios privados. Ese nexo proporciona la plataforma para el ejercicio del poder del Estado localmente.

\section{Burocracia}

La tasa de crecimiento anual de 9-10\% de la economía de la RPCh desde 1978 es mencionada con frecuencia por analistas externos de todo tipo. La media de 6\% anual de tasa de crecimiento alcanzada entre 1952 y 1978 (según las cifras oficiales del Buró Estatal de Estadística de la RPCh), aunque se menciona menos, no es por ello menos significativa, especialmente como base del éxito actual (Buró Estatal de Estadística, 1990: 6). Aunque esta cifra esconde algunas fluctuaciones severas, sobre todo tras las repercusiones del "Gran Salto Adelante", supone que durante los años cincuenta en particular la RPCh estableció un sistema administrativo que funcionaba. Particularmente, la burocracia proporcionaba y dirigía la educación, los servicios de salud y bienestar de acuerdo con los dictados del proceso de planificación estatal. 
Como ya se ha mencionado, el cambio principal en la burocracia con la llegada de la reforma ha sido la introducción de principios de mercado en la distribución de recursos y servicios. Las estructuras administrativas del Estado ahora proporcionan menos bienes y servicios públicos y, en su lugar, desempeñan cada vez más el papel de reguladores del mercado. Los experimentos con la introducción de la Política de Monetarización de la Vivienda en Guiyang en 1998 y posteriormente, diseñados para reformar el mercado de la vivienda, indican el alcance y el impacto de este cambio tanto en la burocracia como en la sociedad en general. La vivienda la proporcionaba cada unidad de trabajo, con una subvención ofrecida por el gobierno local, ya que cada unidad de trabajo era en realidad una rama del Estado, o incluso del propio gobierno local. Había claras desigualdades en este sistema, especialmente porque, aun cuando las empresas en manos del Estado podían albergar a dos tercios de sus empleados de esta manera, sólo a un tercio de los empleados del sector colectivo se les proporcionaba algo similar. Para el año 1998 la inversión estatal en el desarrollo de la vivienda había desaparecido prácticamente de manera local y se había reemplazado por inversiones privadas. Las viviendas subvencionadas por el Estado se vendieron y se terminaron los subsidios, y se reemplazaron por un único subsidio extraordinario para los trabajadores. El gobierno local introdujo políticas diseñadas para diferenciar a los ciudadanos de ingresos altos, que comprarían su propia vivienda, en lugar de recurrir al sistema anterior de vivienda de las unidades de trabajo. Se esperaba que los ciudadanos de ingresos medios o bajos compraran viviendas de bajo costo. Finalmente, los más pobres recibirían viviendas sociales de bajo costo. Los resultados parecen haber incrementado la pobreza urbana, las desigualdades sociales y la aparición de guetos en el mercado de la vivienda de acuerdo con la riqueza (Lee y $\mathrm{Zhu}, 2006$ ).

Una conclusión clara de estos cambios en la burocracia es que el bienestar social pasa cada vez más de las manos de la burocracia del Estado a otras instituciones sociales, que a menudo establecen los habitantes de los pueblos. Oakes detalla las actividades de la $18^{a}$ Asociación del Festival en Jin Family Fort en su descripción de la adopción de estrategias de desarrollo cultural por parte de los pueblos en Guizhou. Por medio de sus actividades variadas y la promoción del turismo, esta asociación recoge suficientes fondos como para sustituir al Estado en la provisión de bienes y servicios públicos básicos (Oakes, 2006). Las investigaciones de Lily Tsai sobre la provisión de bienes públicos en Fujian, Hebei y Jiangxi reflejan hechos similares (Lily Tsai, 2007). 
El impacto de la mercantilización de los servicios públicos en la burocracia local ha sido quizás menos predecible y ciertamente menos intencionado. Como se podría esperar, dados los comentarios anteriores sobre la fuerza creciente de los niveles locales del ejercicio del poder del Estado en su relación con los niveles altos del sistema, la evidencia de estudios locales es que la burocracia local ha caído en manos del gobierno local hasta cierto punto. El análisis de Hsing sobre los nuevos burós de gestión de la tierra y sus actividades es particularmente agudo en este respecto.

La supuesta misión de los burós de la tierra es la conservación de las tierras de labranza. Aunque todavía se encuentran en manos de los dirigentes urbanos del gobierno orientados al crecimiento, los burós locales de gestión de la tierra se han convertido en una vía importante para que el gobierno urbano controle los inventarios de terrenos rurales, supervise la conversión de tierras de labranza y las ventas de arrendamiento de tierras (Hsing, 2006).

La tendencia parecería ser que la burocracia local posiblemente pase a formar parte de la constelación de poder local, controlada por los dirigentes locales. Puede que esto no esté siempre en contra de otras partes de la administración del Estado, pero la posibilidad de que ocurra ese tipo de conflicto es clara, incluso si se contiene dentro del marco del Estado de partido único.

Una observación final sobre las formas en las que la burocracia estatal ya no es lo que era, se puede deducir al considerar la dependencia del origen. La burocracia estatal de la era de la prerreforma ejerció con principios y procedimientos que se cristalizaron a principios de los años cincuenta, pero que se habían heredado de anteriores experiencias del Pcch (Barnett, 1967; Lieberthal y Oksenberg, 1988). Los líderes del PCch dieron por sentada la dependencia del origen de la burocracia antes de 1978, y fue hasta cierto punto una dependencia deseada, representando una forma de fiabilidad. Sin embargo, esto ha cambiado con la llegada de la reforma, donde la burocracia ha necesitado aprender nuevos trucos, no sólo una vez, sino varias, ya que las reformas de hace 20 o 10 años se han tenido que reemplazar. Esto ha provocado que la burocracia haya tenido que desarrollar nuevas estrategias para asegurar que las reformas específicas se implementen y que se mantenga un clima receptivo y productivo de cambio y de mejora. La burocracia se ha tenido que desarrollar a sí misma como una institución que sigue aprendiendo, de maneras que habrían sido completamente extrañas para quienes ocupaban sus oficinas de 1949 a 1978 (Linda Li, 2006b). 


\section{El Estado en transición}

Incluso sin considerar el papel de las interacciones internacionales (el sexto elemento en el modelo mostrado) en el ejercicio del poder del Estado, sería razonable sostener que la evidencia de estudios locales apunta a que en verdad ha habido cambios sustanciales desde 1978. Por supuesto, esto no significa defender que no existen continuidades con la era de la prerreforma, e incluso desde una perspectiva local está claro que estas continuidades ayudan a modelar al Estado y el ejercicio del poder. El liderazgo continúa siendo crucial en el funcionamiento de la RPCh como Estado de partido único, y los nombramientos de los dirigentes, a pesar de muchos cambios organizativos, continúan siendo un mecanismo de control del PCch. Incluso a pesar del aparente cambio de valores, todavía existe autoridad en el pensamiento marxistaleninista de Mao Zedong como ideología del Estado de partido único. Aunque puede no haber llevado a la asignación de fondos que requería el programa, está claro que una gran parte de la justificación de la campaña de "Apertura a Occidente" que comenzó en 2000 fue precisamente un llamamiento tanto a las consideraciones generales de igualdad como a las promesas anteriores hechas para desarrollar la Región Occidental (Goodman, 2004b).

Al considerar a la RPCh como un Estado en transición, hay dos preguntas que se hacen con frecuencia, a las que se debería añadir una tercera: ¿debería cambiar el Estado incluso de manera más fundamental? ¿Cambiará? ¿Podrá hacerlo? A modo de conclusión, sería útil considerar algunas de las posibles respuestas.

Existen bastantes estudios que indican que el Estado debería cambiar incluso más fundamentalmente, algunos de los cuales se han reflejado en argumentos que se han presentado aquí. Aunque es difícil evitar un matiz moral en esas perspectivas, quienes defienden el cambio no lo hacen todos basándose en que China debería parecerse más a Estados Unidos o en que un Estado de partido único comunista sea indeseable. Hay también un argumento muy claro entre los presentados que explica que el ejercicio del poder estatal en China sería más eficiente y eficaz a la hora de proporcionar bienes públicos, si rindiera más cuentas y fuera más representativo.

La pregunta que se hace con frecuencia, “¿cambiará la RPCh?”, quiere decir normalmente “ ¿dejará de ser el Pcch el partido dirigente?”, y se acompaña con una segunda pregunta sobre cuándo sucederá. Los analistas sociales, que suelen ser sensatos, rechazan hacer predicciones tan precisas, o al menos 
muchos de ellos así lo hacen. Una excepción notable, que puede hablar del caso de la RPch, al menos en parte, es el proyecto de investigación llevado a cabo bajo la dirección de Jack Goldstone durante los últimos años de la década de los ochenta, que investigaba revoluciones contemporáneas. Este proyecto concluyó, inter alia, que no se llegaba a una situación revolucionaria hasta que coincidían tres condiciones en forma simultánea: una crisis de Estado, una división de la élite y la movilización de las masas (Gurr y Goldstone, 1991). Aunque la atención de muchos fuera de las fronteras de la RPCh se dirige a los ejemplos de disturbios de masas localizados y a pequeña escala, el propio PCCh ha entendido claramente la importancia de evitar divisiones públicas de su dirección, especialmente tras los sucesos de mayo de 1989.

Por último, hay una pregunta que se considera con menos frecuencia y es si el Estado realmente puede cambiar. Por supuesto, puede haber un cambio fundamental, pero no necesariamente sin un cataclismo o dentro de las estructuras establecidas para el ejercicio del poder del Estado. Ésta es la pregunta más intrigante de las que se hacen, desde la perspectiva de entender el Estado como un constructo político. La justificación de la RPch radica tanto en el nacionalismo chino moderno como en las configuraciones del pensamiento marxista-leninista de Mao Zedong. De momento, ambos se igualan. La experiencia de Estados que otrora fueron de partido único comunistas sugiere que es difícil, si no imposible, que un Estado de partido único evolucione y se convierta en algo diferente, aunque debe haber un elemento de dependencia de origen en el posterior ejercicio del poder estatal.

Desde la perspectiva local, durante las tres últimas décadas el Estado se ha centrado en el nacionalismo y en la creación de riqueza. El sistema gubernamental se ha descentralizado mucho con el ejercicio del poder estatal, quedando en gran medida en manos de la comunidad de interés que se ha creado entre los cuadros locales y los empresarios. Hay poca evidencia que indique que el cambio fundamental es inminente, sobre todo porque el Estado de partido único ha mostrado una gran capacidad de adaptación a los desarrollos socioeconómicos de los últimos 30 años. Por otro lado, si intervinieran otros factores serían posibles soluciones federales, como también la aún gran diferenciación local de la política y el ejercicio del poder del Estado. mi 


\section{Referencias bibliográficas}

Barnett, A. Doak, Cadres, Bureaucracy, and Political Power in Communist China, Columbia University Press, 1967.

Blecher, Marc, y Vivienne Shue, Tethered Deer: Government and Economy in a Chinese County, Stanford University Press, 1996.

Carrillo, Beatriz, "From coal black to hospital white: New welfare entrepreneurs and the pursuit of a cleaner status", en David S. G. Goodman (ed.), The New Rich in China: Future Rulers, Present Lives, Londres, Routledge, 2008.

Chen, Chih-jou Jay, Transforming Rural China: How Local Institutions Shape Property Rights in China, Londres, Routledge, 2004.

Chen, Minglu, "Entrepreneurial women: personal wealth, local politics and tradition", en David S. G. Goodman (ed.), The New Rich in China: Future Rulers, Present Lives, Londres, Routledge, 2008.

Chung, Jae Ho (ed.), Cities in China: Recipes for Economic Development in the Reform Era, Londres, Routledge, 1999.

- - "Studies of central-provincial relations in the People's Republic of China: A mid-tem appraisal', The China Quarterly, núm. 142, 1995.

- - Central Control and Local Discretion in China: Leadership and Implementation during Post-Mao Decollectivization, Oxford University Press, Oxford, 2000.

Dickson, Bruce J., Red Capitalists in China: The Party, Private Entrepreneurs, and Prospects for Political Change, Cambridge University Press, Cambridge, 2003.

- - Wealth into Power: The Communist Party's Embrace of China's Private Sector, Cambridge University Press, Cambridge, 2008.

Duckett, Jane, The Entrepreneurial State in China: Real Estate and Commerce Departments in Reform Era Tianjin, Routledge, Londres, 1998.

Duckett, Jane, y Beatriz Carrillo (eds.), China's Social Welfare Mix: Conceptualizing State and Societal Responses to Evolving Social Problems, Routledge, Londres, 2009.

Economy, Elizabeth C., y Adam Segal, "The G-2 Mirage: Why the United States and China are not ready to Upgrade Ties", Foreign Affairs, mayo-junio de 2009.

Edwards, Louise, "Diversity and evolution in the state-in-society: International influences in combating violence against women", en Linda Chelan Li 
(ed.), The Chinese State in Transition: Protests and Contests in Local China, Routledge, Londres, 2009.

Fitzgerald, John (ed.), Rethinking China's Provinces, Routledge, Londres, 2002.

Friedmann, John, "The world city hypothesis", Development and Change, vol. 17, 1986.

Gong, Ting, "Corruption and local governance: The double identity of Chinese local governments in market reform", Pacific Review, vol. 19, núm. 1, 2006.

Gong, Ting, y Chen Feng, "Institutional reorganization and its impact on decentralization", en Jia Hao, y Lin Zhimin (eds.), Changing Central-Local Relations in China: Reform and State Capacity, Boulder, Westview Press, 1994.

Goodman, David S. G., "The Chinese political order after Mao: 'Socialist Democracy' and the exercise of state power”, Political Studies, vol. 33, núm. 2, junio de 1985.

- - China's Provinces in Reform: Class, Community and Political Culture, Routledge, Londres, 1997.

- - "Structuring local identity: Nation, province and county in Shanxi during the 1990s", The China Quarterly, núm. 172, diciembre de 2002.

- - "Localism and entrepreneurship: History, identity and solidarity as factors of production", en Barbara Krug (ed.), China's Rational Entrepreneurs: The Development of the New Private Business Sector, Routledge, Londres, 2004a.

- - (ed.), China's Campaign to "Open Up the West": National, Provincial and Local Perspectives, Cambridge University Press, Cambridge, 2004b.

- - (ed.), The New Rich in China: Future Rulers, Present Lives, Routledge, Londres, 2008a.

- - (ed.), "Why China has no new middle class: Cadres, managers, and entrepreneurs", en David S. G. Goodman (ed.), The New Rich in China: Future Rulers, Present Lives, Routledge, Londres, 2008b.

Groenewold, Nicolaas, Anping Chen, y Guoping Lee, Linkages Between China's Regions: Measurement and Policy, Edward Elgar, Cheltenham, Reino Unido, 2008.

Guo, Yingjie, Cultural Nationalism in Contemporary China: The Search for National Identity under Reform, Routledge, Londres, 2004. 
Gurr, Ted Robert, y Jack A. Goldstone, "Comparisons and policy implications", en Jack A. Goldstone, Ted Robert Gurr, y Farrokh Moshiri (eds.), Revolutions of the Late Twentieth Century, Westview Press, Boulder, 1991.

Hartshorne, Richard, "The Functional Approach in Political Geography", Annals of the Association of American Geographers, vol. 40, 1950.

Harvey, David, A Brief History of Neoliberalism, Oxford University Press, Oxford, 2005.

Hendrischke, Hans, y Feng Chongyi (eds.), The Political Economy of China's Provinces: Comparative and Competitive Advantage, Routledge, Londres, 1999.

Hsing, You-tien, "Brokering power and property in China's townships", Pacific Review, vol. 19, núm. 1, 2006.

$\mathrm{Hu}$, Fuguo, "Renminde shengchang yao dui renmin fuze ('The people's governor must be responsible to the people')", en Hu Fuguo, Jiang zhenhua, ban shishi, zuo biaoshuai: zaichuang Sanjin huihuang (Decir la verdad, hacer que sucedan las cosas y poner el ejemplo: Reconstruyendo la gloria de Shanxi), Zhonggong zhongyang dangxiao chubanshe, Pekín, 1996.

Huang, Yasheng, Capitalism with Chinese Characteristics: Entrepreneurship and the State, Cambridge University Press, Cambridge 2008.

Ionescu, Ghita, The Politics of the Eastern European Communist Party States, Weidenfeld and Nicholson, Londres, 1967.

Jia Hao, y Lin Zhimin (eds.), Changing Central-Local Relations in China: Reform and State Capacity, Westview Press, Boulder, 1994.

Jiang Zemin, "Report to $16^{\text {th }}$ Congress of CCP", Xinhua (New China News Agency, English), 8 de noviembre de 2002.

Jonathan, Unger, y Anita Chan, "Inheritors of the boom: private enterprise and the role of local government in a rural south China township", The China Journal, vol. 42, 1999.

Keng, C. W. Kenneth, “China's Future Economic Regionalization”, Journal of Contemporary China, vol. 10, núm. 29, noviembre de 2001.

Landry, Pierre F., Decentralized Authoritarianism in China: The Communist Party's Control of Local Elites in the Post-Mao Era, Cambridge University Press, Cambridge, 2008.

Lee, James, y Zhu Ya Peng, "Urban governance, neoliberalism and housing reform in China", en Linda Chelan Li (ed.), The Chinese State in Transition, Routledge, Londres, 2009. 
Li, Linda Chelan, Centre and Provinces: China, 1978-1993, Power as Non-Zero Sum, Oxford University Press, Oxford, 1998.

- - "The state in change: Processes and contestations in local China: An introduction”, Pacific Review, vol. 19, núm. 1, marzo, 2006a.

- - "Embedded institutionalization: Sustaining the rural tax reform in China”, Pacific Review, vol. 19, núm. 1, marzo, 2006b.

- - The Chinese State in Transition: Protests and Contests in Local China, Routledge, Londres, 2009.

Lieberthal, Kenneth, y Michel Oksenberg, Policy Making in China: Leaders, Structures and Processes, Princeton University Press, Princeton, 1988.

MacFarquhar, Roderick, "The succession to Mao and the end of Maoism", en Roderick MacFarquhar, y John K. Fairbank, The Cambridge History of China, vol. 15, Part 2, Revolutions within the Chinese Revolution 1966-1982, Cambridge University Press, Cambridge 1991.

Maddison, Angus, Chinese Economic Performance in the Long Run, $2^{\mathrm{a}}$ edición, OCDE, 2007.

Mann, James, The China Fantasy, Viking, Nueva York, 2007.

McFaul, Michael, "Russia's rough ride", en Larry Diamond, Marc F. Plattner, Yun-han Chu, y Hung-mao Tien (eds.), Consolidating the Third Wave Democracies: Regional Challenges, John Hopkins University Press, 1997.

Mertha, Andrew, China's Water Warriors: Citizen Action and Policy Change, Cornell University Press, 2008.

Migdal, Joel S., State in Society: Studying How States and Societies Transform and Constitute One another, Cambridge University Press, 2001.

Montinola, Gabriella, Tingyi Qian, y Barry R. Weingast, "Federalism, Chinese Style: The Political Basis for Economic Success in China", World Politics, vol. 48, octoubre de 1995.

Naughton, Barry J., y Dali L. Yang (eds.), Holding China Together: Diversity and National Integration in the Post-Deng Era, Cambridge University Press, Cambridge, 2004.

O’Brien, Kevin J. (ed.), Popular Protest in China, Harvard University Press, Harvard, 2008.

Oakes, Tim, "China's provincial identities: Reviving regionalism and reinventing 'Chineseness"', The Journal of Asian Studies, vol. 59, núm. 3, agosto de 2000.

_- "Cultural strategies of development: Implications for village governance in China”, Pacific Review, vol. 19, núm. 1, marzo de 2006. 
Oakes, Tim, y Louisa Schein (eds.), Translocal China: Linkages, Identities and the Reimagining of Space, Routledge, Londres, 2006.

Oi, Jean C., State and Peasant in Contemporary China: The political economy of village government, University of California Press, Berkeley, 1989.

- - "Fiscal reform and the origins of local state corporatism in China", World Politics, núm. 45, 1992.

- - "The role of the local state in China's transitional economy", The China Quarterly, núm. 144, diciembre de 1995.

- - Rural China Takes Off: Institutional Foundations of Economic Reform, University of California Press, Berkeley, 1999.

Pei, Minxin, China's Trapped Transition: The Limits of Developmental Autocracy, Harvard University Press, Harvard, 2006.

Perry, Elizabeth J., "Studying Chinese politics: Farewell to revolution?", The China Journal, núm. 57, enero de 2007.

Perry, Elizabeth J., y Mark Selden (eds.), Chinese Society: Change, Conflict and Resistance, Routledge, Londres, 2000.

Scissors, Derek, "Deng undone: The costs of halting market reform in China", Foreign Affairs, mayo-junio de 2009.

Scmp, Us Survey of the China Mainland Press, núm. 1850, 10 de septiembre de 1958.

Solinger, Dorothy J., "Economic reform via reformulation: Where do rightists ideas come from?", en M. E. Sharpe, China's Transition from Socialism: Statist Legacies and Market Reforms, 1980-1990, Nueva York, 1993.

State Statistical Bureau, Lishi tongji ziliao huibian 1949-1989, (Collection of Historical Statistical Materials 1949-1989), Zhongguo tongji chubanshe, Pekín, 1990.

Teiwes, Frederick C., "The purge of provincial leaders, 1957-1958”, The China Quarterly, núm. 27, Julio de 1966.

Tomba, Luigi, "The forest city: Homeownership and new wealth in Shenyang", en David S. G. Goodman (ed.) The New Rich in China: Future Rulers, Present Lives, Routledge, Londres, 2008.

Tsai, Kellee S., Capitalism without Democracy: The Private Sector in Contemporary China, Cornell University Press, Cornell, 2007.

Tsai, Lily L., Accountability without Democracy: Solidary Groups and Public Goods Provision in Rural China, Cambridge University Press, Cambridge, 2007. 
Walder, Andrew G. (ed.), Zouping in Transition: The Process of Reform in Rural China, Harvard University Press, Harvard, 1998.

Wang, Jing (ed.), Locating China: Space, Place and Popular Culture, Routledge, Londres, 2005.

Weber, Max, The Theory of Social and Economic Organization, Free Press, Glencoe, 1997.

Weng, Naiqun, "Flows of heroin, people, capital, imagination, and the spread of HIV in southwest China", en Tim Oakes, y Louisa Schein (eds.), Translocal China: Linkages, Identities and the Reimagining of Space, Routledge, Londres, 2006.

Wertheim, W. F., Evolution and Revolution, Penguin, Harmondsworth, 1974. White, Gordon, "Prospects for civil society", en David S. G. Goodman, y Beverley Hooper (eds.), China's Quiet Revolution, Longman Cheshire, Melbourne, 1994.

White, Gordon, The Politics of Class and Class Origin, Australian National University Press, Canberra, 1976.

Whiting, Susan H., Power and Wealth in Rural China: The political Economy of Institutional Change, Cambridge University Press, Cambridge, 2001.

Yep, Ray, Manager Empowerment in China: Political Implications of Rural Industrialization in the Reform Era, Routledge, Londres, 2003. 